\title{
Delayed Spinal Epidural Hematoma after En Block Spondylectomy for Vertebral Ewing's Sarcoma
}

\author{
Boram Kim¹, Seong-Hwan Moon¹, Sun-Yong Kim¹, Ho-Joong Kim², Hwan-Mo Lee \\ ${ }^{1}$ Department of Orthopedic Surgery, Yonsei University College of Medicine, Seoul, Korea \\ ${ }^{2}$ Yonsei Sarang Hospital, Seoul, Korea
}

\begin{abstract}
We report here on a case of a 23-year-old male who received en block spondylectomy for a vertebral Ewing's sarcoma at our hospital. Nine days after surgery, he presented with severe back pain and motor weakness of the lower extremities. Based on the physical examination and the computed tomography scan, he was diagnosed with acute cauda equina syndrome that was caused by compression from an epidural hematoma. His neurological functions recovered after emergency evacuation of the hematoma. This case showed that extensive surgery for a malignant vertebral tumor has a potential risk of delayed epidural hematoma and acute cauda equina syndrome and this should be treated with emergency evacuation.
\end{abstract}

Key Words: Epidural hematoma, Ewing's sarcoma, Spine, En block spondylectomy

\section{Introduction}

Symptomatic spinal epidural hematoma is an infrequent complication of spinal surgery. Spinal epidural hematomas usually occur within 24 hours after surgery [1]. Delayed epidural hematoma is defined as those occurring 3 days after surgery. Although delayed epidural hematomas are very rare, they can occur even 1 week after surgery [1-4].

Operating on malignant sarcomas in the vertebra requires extensive procedures and preoperative chemotherapy which can cause massive bleeding and a tendency to bleed. Although spinal surgery for a malignant vertebral tumor presents the potential risks of postoperative bleeding and hematomas, there have been no reports of delayed onset epidural hematomas as well as epidural hematomas after extensive surgery for a malignant vertebral tumor.

We report here on a postoperative epidural hematoma that occurred 9 days after performing en bloc spondylectomy of the L3 vertebra because of Ewing's sarcoma.

\section{Case Report}

A 23-year-old male presented with radiating pain in his left lower extremity and lower back. The back pain and radiating pain had started 3 months previously and they had become aggravated for the previous 1 month, and especially at night. He had no past medical or surgical history.

There were no remarkable findings except for dyesthesia on the left L3 dermatome on the physical examination. Although simple radiographs of the lumbar spine showed unremarkable findings, magnetic resonance imaging (MRI) revealed intraosseous mass lesions involving the left side of the vertebral body, the pedicle and the transverse process of the L3. The mass extended outside the vertebra and involved the paraspinal muscle close to the left transverse process (Fig. 1). Computed tomography (CT)-guided needle biopsy showed malignant small round cell tumor that was

Received Feb 8, 2010; 1st Revised Mar 15, 2010; Accepted Mar 18, 2010

Corresponding author: Hwan-Mo Lee, MD

Department of Orthopedic Surgery, Yonsei University College of Medicine,

134 Sinchon-dong, Seodaemun-gu, Seoul 120-752, Korea

Tel: +82-2-2228-2191, Fax: +82-2-363-1139, E-mail: hwanlee@yuhs.ac 
compatible with Ewing's sarcoma.

The patient received three cycles of preoperative chemotherapy. The chemotherapeutic regimen included doxorubicin, ifosfamide and etoposide phosphate. The MRI after the preoperative chemotherapy showed the size of the paraspinal mass had markedly decreased compared with that of the previous study (Fig. 2).

En bloc spondylectomy was performed using both the posterior and anterior approaches. All the posterior elements of L3 were exposed and the transverse processes were first cut off during the posterior approach. The L3 isthmus was severed and the spinous process, lamina and inferior articular processes were removed together. The residual posterior parts, including the superior articular processes, were all removed. The cut surfaces of the pedicles were sealed with bone wax. Posterior instrumentation and posterolateral auto-iliac bone grafts were performed between $\mathrm{L} 1$ and $\mathrm{L} 4$.

The anterolateral retroperitoneal approach was performed with the patient in the right semilateral decubitus position. The vertebral body was removed after blunt dissection of the soft tissues around the L3 body and excisions of the L2/3 and L3/4 intervertebral discs. Double barreled $6 \mathrm{~cm}$ strut grafts, which were longitudinally obtained from the left auto-fibular bone, were inserted and these replaced the L3 body (Fig. 3).

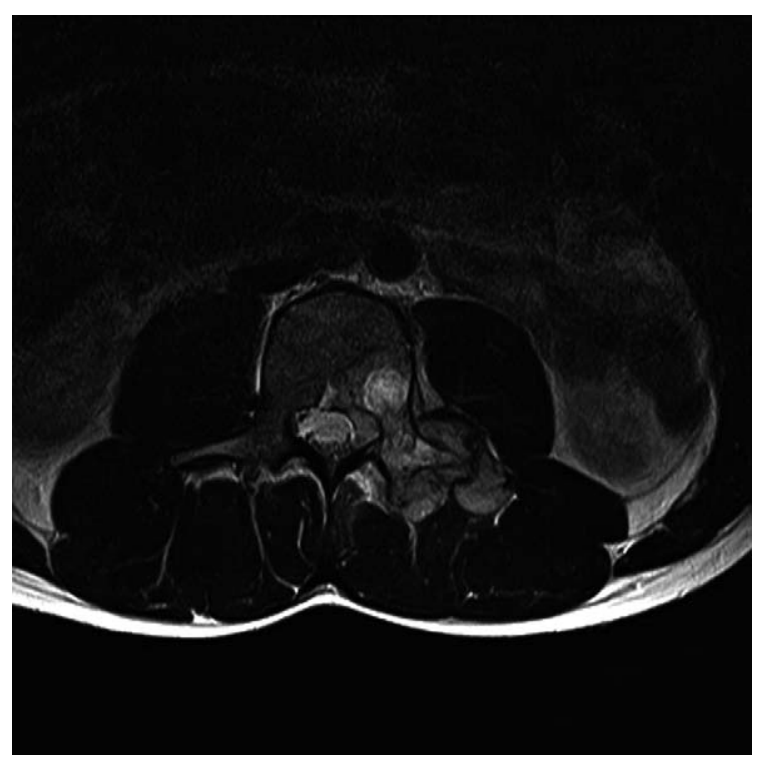

Fig. 1. The initial axial T2-weighted magnetic resonance imaging shows that the tumor mass involved the left L3 vertebral body, pedicle and transverse process. The mass extended outside the vertebra and it involved the paraspinal muscle close to the left transverse process.
The intraoperative bleeding from the epidural veins and segmental vessels was controlled by electrical coagulation or ligation of the left segmental vessels. The right segmental vessels could not be approached and they were not clearly identified even after removing the L3 body. But all the bleeders were controlled by electrical coagulation and compression. Closed suction drains were placed deep to the fascia anteriorly and posteriorly. The patient showed no abnormal neurological findings postoperatively.

The preoperative hemoglobin, hematocrit, platelet count, prothrombin time and thrombin time were all normal. There was $6,330 \mathrm{ml}$ of blood loss during the operation, which took 11 hour and 20 minutes. Two thousand and four hundred $\mathrm{ml}$ of packed red blood cells and $700 \mathrm{ml}$ fresh frozen plasma were infused during surgery. The hemoglobin level was 9.9 $\mathrm{g} / \mathrm{dl}$, the platelet count was $100 \times 10^{3}$ and the prothrombin time and thrombin time were within the normal ranges in the immediate postoperative period.

During the first 3 postoperative days, $1,580 \mathrm{ml}$ were drained from the main operative site, and then this began to decrease and the drains were removed on the 5th postoperative day.

The patient was making good progress and he could tolerate sitting without a brace, but standing was not permitted. Nine days after the operation, the patient complained of sudden right flank pain and lower back pain during position

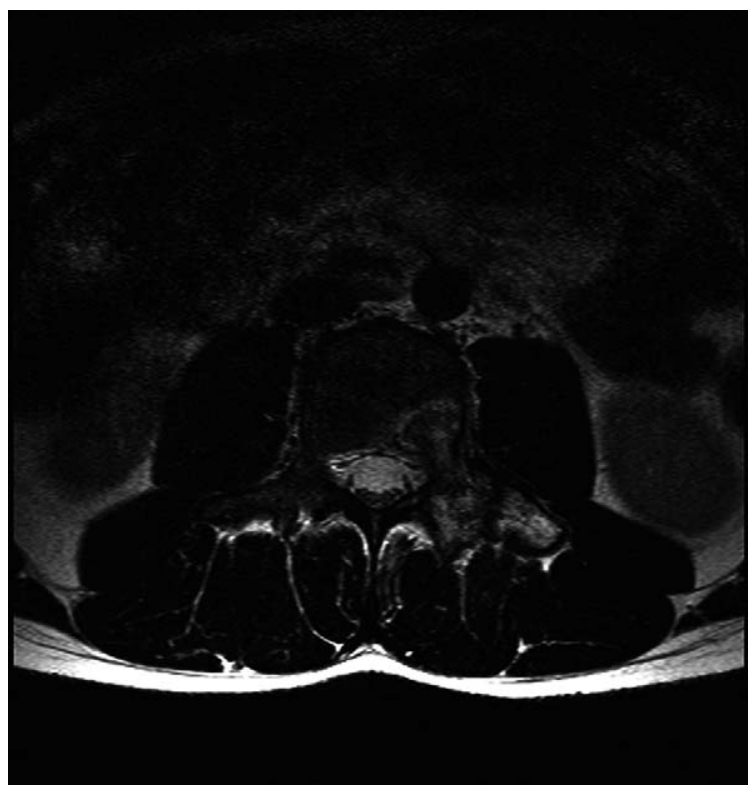

Fig. 2. The axial T2-weighted magnetic resonance imaging after pre-operative chemotherapy shows a marked decrease in the size of the paraspinal mass close to the left transverse process. 
change. There had been no trauma or strain before the pain had begun. At that time the patient showed no neurological abnormalities, but a sudden sensory loss below the L4 dermatome and motor weakness in the lower extremities were noted 2 hours later.
Neurological examination revealed that the ankle dorsiflexion, great toe extension and ankle plantar flexion motor grades had decreased to G0 bilaterally. A sensory test showed anesthesia below the L4 dermatomes in both lower extremities and saddle anesthesia. Swelling on the operative
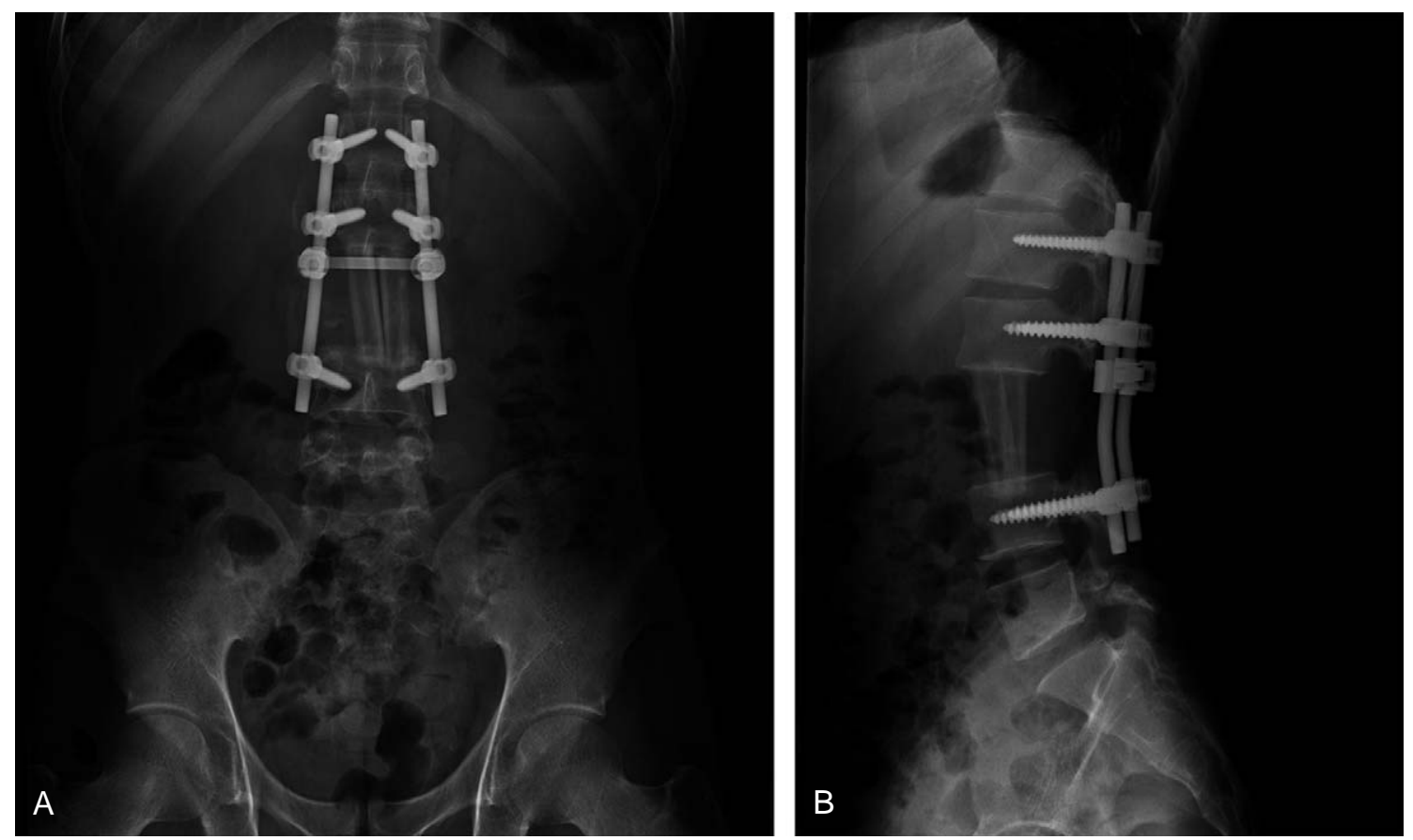

Fig. 3. The postoperative lumbar anteroposterior (A) and lateral (B) X-rays shows the strut bone grafts replacing the L3 body after spondylectomy.
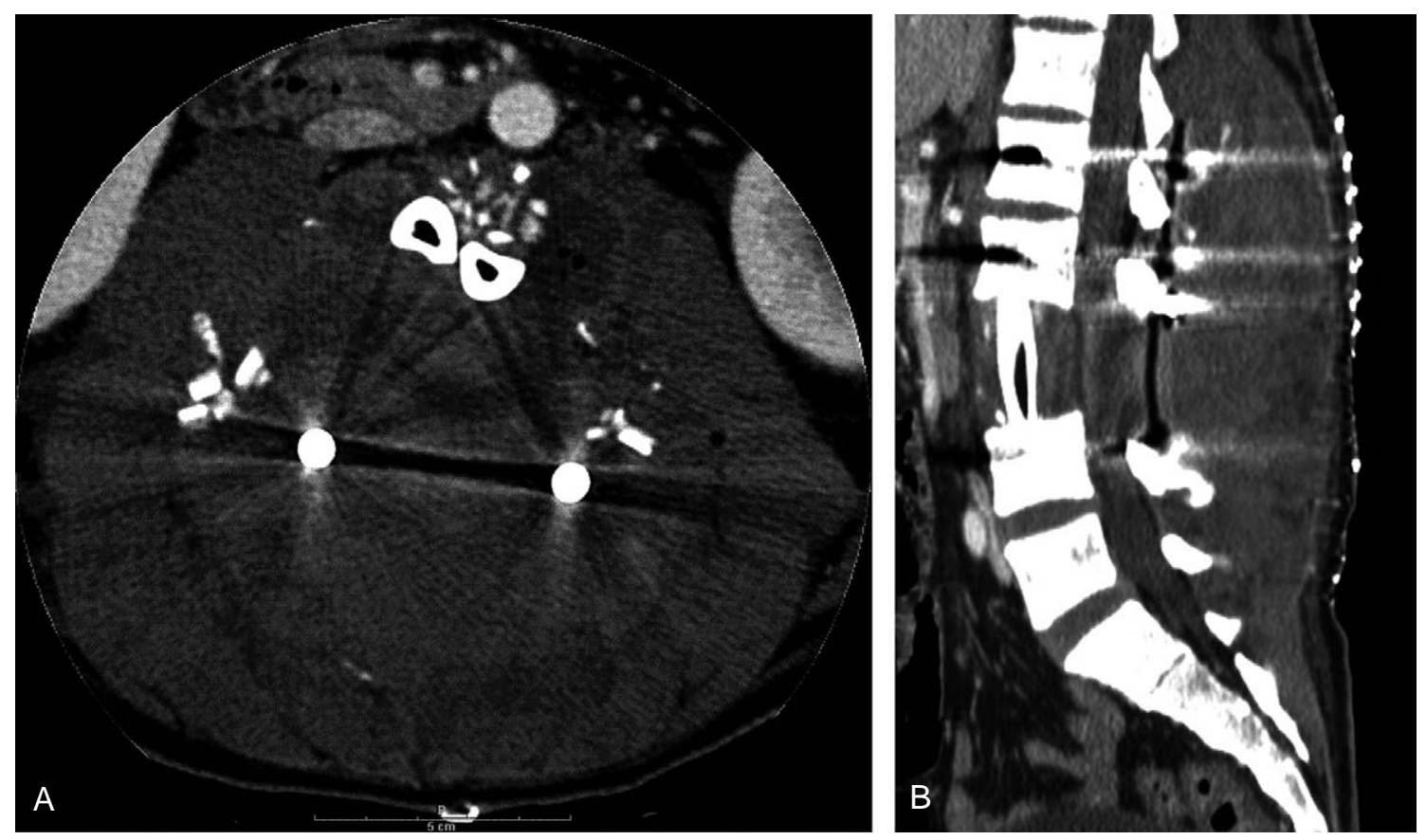

Fig. 4. The axial (A) and reconstructed sagittal images (B) of a computed tomography scan show a lens shaped, huge hematoma bulging the skin. 
site was also noted together with marked tenderness. CT scans of the lumbar spine showed a huge hematoma compressing the dural sac (Fig. 4). The hemoglobin level was $11.0 \mathrm{~g} / \mathrm{dl}$, the platelet count was $284 \times 10^{3}$ and the prothrombin time and thrombin time were within the normal ranges at that time.

An emergency operation was performed 5 hours after the symptoms started. A huge hematoma that occupied dead space was noted after incision. After evacuating the hematoma, the dural sac was identified and a small bleeding point in the paraspinal muscles was noted at the left L2 level. The bleeding point was so small that we could not determine whether it was arterial or venous bleeding. However, it was not from the segmental artery. After evacuation, the wound was closed with leaving a suction drain.

The patient's pain subsided immediately after the operation. The motor and sensory functions in both lower extremities fully recovered in the immediate postoperative period. The remainder of the patient's course was uncomplicated. Adjuvant chemotherapy was started 3 weeks after the second operation.

\section{Discussion}

Symptomatic spinal epidural hematoma is an uncommon complication after spinal surgery and the incidence of this was reported to be $0.1 \%$ to $0.24 \%$ [1,5-7]. Although most spinal epidural hematomas occur within 24 hours after surgery, delayed hematomas can also occur.

Uribe et al. [1] defined delayed postoperative epidural hematoma as that occurring 3 days after surgery. We found several reports of delayed postoperative epidural hematomas of the spine in a search of the medical literature. Spanier and Stambough [4] reported on delayed postoperative epidural hematomas that caused acute cauda equina syndrome 16 days after lumbar decompression and fusion in 2000. Uribe et al. [1] reported on 7 cases of delayed epidural hematoma after spinal surgery and Neo et al. reported on a case that presented 9 days after laminoplasty [2]. In 2006, Sokolowski et al. [3] reported on 4 cases after lumbar surgery. Although there have been a few reports of delayed epidural hematoma after common spinal procedures, no cases have been reported after extensive surgery such as en block spondylectomy for a malignant vertebral tumor. This is the first case report of a delayed postoperative epidural hematoma after total spondylectomy for treating a malignant vertebral tumor.
Bleeding in the vertebral venous plexus has been reported as the cause of spinal epidural hematoma, including delayed onset spinal epidural hematoma [1,5,8,9]. Delayed bleeding can occur as a result of arterial tears, which are caused by paraspinal muscle strain [2]. In this case, we did not discover whether the bleeding originated from a vein or artery. It was most likely that the muscular branch of the venous plexus caused the delayed hematoma.

The classic presentation of spinal epidural hematomas is severe back pain followed by radiating pain and finally motor weakness $[1,7]$. This patient displayed severe flank pain and lower back pain prior to motor weakness, but there was no radiating pain between those symptoms.

Postoperative spinal hematomas can compress the spinal cord or cauda equina. Immediate evacuation of the hematoma is needed for the cases of a compressed spinal cord. But there have been conflicting results regarding acute cauda equina syndrome. Delamarter et al. [10] demonstrated no significant difference between early decompression and late decompression in animal studies. However, the clinical cases showed that early decompression could achieve better results $[1,3,6,7]$. After we evacuated the hematoma for acute cauda equina syndrome, which was caused by a delayed hematoma, the patient's neurological functions fully recovered. So, this case validates that immediate decompression of acute cauda equina syndrome caused by hematoma achieves a good outcome.

There have been several studies that reviewed the risk factors for postoperative epidural hematomas of the spine $[3,5,6,8]$. The preoperative risk factors included old age [5], coagulopathy [8], high international normalized ratio [5,11] and the use of non-steroidal anti-inflammatory drugs [5]. Multilevel procedures and a blood loss more than $1,000 \mathrm{ml}$ were reported as intraoperative risk factors [5,8].

Operating on malignant vertebral tumors poses a potential risk for incurring postoperative epidural hematomas. Patients with malignant spine tumor such as Ewing's sarcoma and osteosarcoma usually undergo preoperative neoadjuvant chemotherapies to decrease the mass and prevent micrometastasis $[12,13]$. This can cause myelosupression, and especially for the regimens that include doxorubicin [14]. If the bone marrow is suppressed, the function and number of platelets could be affected, which it might cause coagulopathy, although the perioperative platelet count, and the prothrombin time and thrombin time of our patient were within the normal ranges. Extensive dissection, which can cause massive blood loss, is needed for cases of en bloc 
spondylectomy for definite local control of malignant tumor $[15,16]$. In addition, a huge dead space remained after surgery. En block spondylectomy, as well as multilevel surgery, might increase the risk of a postoperative hematoma.

In conclusion, surgeons should be vigilant for postoperative epidural hematomas, which can occur even a week and more after surgery, when performing an extensive procedure such as en bloc spondylectomy for malignant vertebral tumors. If postoperative epidural hematoma does occur, then we recommend immediate evacuation.

\section{REFERENCES}

1. Uribe J, Moza K, Jimenez O, Green B, Levi AD. Delayed postoperative spinal epidural hematomas. Spine J 2003; 3:125-9.

2. Neo M, Sakamoto T, Fujibayashi S, Nakamura T. Delayed postoperative spinal epidural hematoma causing tetraplegia: case report. J Neurosurg Spine 2006;5:251-3.

3. Sokolowski MJ, Dolan M, Aminian A, Haak MH, Schafer MF. Delayed epidural hematoma after spinal surgery: a report of 4 cases. J Spinal Disord Tech 2006;19:603-6.

4. Spanier DE, Stambough JL. Delayed postoperative epidural hematoma formation after heparinization in lumbar spinal surgery. J Spinal Disord 2000;13:46-9.

5. Awad JN, Kebaish KM, Donigan J, Cohen DB, Kostuik JP. Analysis of the risk factors for the development of postoperative spinal epidural haematoma. J Bone Joint Surg Br 2005;87:1248-52.

6. Yi S, Yoon do H, Kim KN, Kim SH, Shin HC. Postoperative spinal epidural hematoma: risk factor and clinical outcome. Yonsei Med J 2006;47:326-32.

7. Lawton MT, Porter RW, Heiserman JE, Jacobowitz R, Sonntag VK, Dickman CA. Surgical management of spinal epidural hematoma: relationship between surgical timing and neurological outcome. J Neurosurg 1995;83:1-7.

8. Kou J, Fischgrund J, Biddinger A, Herkowitz H. Risk factors for spinal epidural hematoma after spinal surgery. Spine (Phila Pa 1976) 2002;27:1670-3.

9. Groen RJ, Ponssen H. The spontaneous spinal epidural hematoma: a study of the etiology. J Neurol Sci 1990;98: 121-38.

10. Delamarter RB, Sherman JE, Carr JB. 1991 Volvo Award in experimental studies. Cauda equina syndrome: neurologic recovery following immediate, early, or late decompression. Spine (Phila Pa 1976) 1991;16:1022-9.

11. Sokolowski MJ, Garvey TA, Perl J 2nd, et al. Prospective study of postoperative lumbar epidural hematoma: incidence and risk factors. Spine (Phila Pa 1976) 2008;33:10813.

12. Wang VY, Potts M, Chou D. Sarcoma and the spinal column. Neurosurg Clin N Am 2008;19:71-80.

13. Ludwig JA. Ewing sarcoma: historical perspectives, current state-of-the-art, and opportunities for targeted therapy in the future. Curr Opin Oncol 2008;20:412-8.

14. Tascilar M, Loos WJ, Seynaeve C, Verweij J, Sleijfer S. The pharmacologic basis of ifosfamide use in adult patients with advanced soft tissue sarcomas. Oncologist 2007;12: 1351-60.

15. Tomita K, Kawahara N, Baba H, Tsuchiya H, Fujita T, Toribatake Y. Total en bloc spondylectomy: a new surgical technique for primary malignant vertebral tumors. Spine (Phila Pa 1976) 1997;22:324-33.

16. Samartzis D, Marco RA, Benjamin R, Vaporciyan A, Rhines LD. Multilevel en bloc spondylectomy and chest wall excision via a simultaneous anterior and posterior approach for Ewing sarcoma. Spine (Phila Pa 1976) 2005; 30:831-7. 